\title{
Roux-en-Y Gastric Bypass Improves Hepatic Glucose Metabolism Involving Upregulation of Sirtl in Type 2 Diabetes Mellitus
}

\author{
Chunjie Su' \\ Qian Cheng ${ }^{2}$ \\ Liyun Wang ${ }^{2}$
}

'Department of Gastrointestinal Surgery, jingmen No.I People's Hospital, Jingmen, 448000, People's Republic of China; ${ }^{2}$ Department of Endocrinology, Yixing People's Hospital, Yixing, 214200, Jiangsu, People's Republic of China
Correspondence: Liyun Wang Department of Endocrinology, Yixing People's Hospital, No. 75, Tongzhenguan Road, Yicheng Street, Yixing, 214200 , Jiangsu, People's Republic of China Email wangliyunly@I63.com
Background: Roux-en-Y gastric bypass (RYGB) is the most effective treatment for type 2 diabetes mellitus (T2DM). Previous studies have reported that silent information regulator 1 (Sirt1) closely relates to many pathological processes of glucose metabolism and insulin resistance (IR). However, it is unclear whether Sirt1 is involved in the hepatic glucose metabolism of T2DM after RYGB.

Methods: T2DM rats were randomly divided into four groups: Control, DM, Diet and RYGB. Normal rats were served as the control group. Hematoxylin and eosin (H\&E) staining and Masson staining assays were performed to explore the changes of liver fibrous tissue after RYGB. The effect of RYGB on the protein expression of Sirtl was detected by the Western blotting assay and immunohistochemical assay. Next, we built the insulin resistance model of human hepatocyte cell lines (FL62891 and HHL5) using the human recombinant insulin. Western blotting assay was applied to determine the expression of Sirt1 and the expression change of IRS1/mTOR2 /PKB pathway-related proteins in FL62891 and HHL5 cells. Additionally, the effects of Sirt1 on the expression of PTP1B and FGF-21 in insulin-resistant FL62891 and HHL5 cells were investigated using Western blotting and immunofluorescence assay.

Results: Our results showed that following RYGB improved the pathological changes of liver and increased the expression of Sirt1 in rats with T2DM compared with the diabetic rats. In experiments in vitro, the expression of Sirt1 was downregulated in insulin-resistance FL62891 and HHL5 cells. Moreover, overexpression of Sirt1 significantly increased the expression of FGF-21 whereas decreased the expression of PTP1B in insulin-resistance FL62891 and HHL5 cells. These above changes were alleviated in RYGB and Diet groups. Furthermore, RYGB could improve the glucose metabolism through activating IRS1/ mTOR2/PKB pathways by regulating Sirt1 in rats with T2DM.

Conclusion: RYGB could significantly improve hepatic glucose metabolism and increase the expression of Sirt1 in T2DM rats, which is related to the IRS1/mTOR2 /PKB pathway. Keywords: Roux-en-Y gastric bypass, RYGB, hepatic glucose metabolism, insulin resistance, Sirt1, IRS1, mTOR2, PKB

\section{Introduction}

Diabetes mellitus (DM) is a global and national health concern with high significance, which is also the major challenge for patients with DM. ${ }^{1}$ It is the world's ninth leading cause of death. ${ }^{2}$ Noticeably, type 2 diabetes mellitus (T2DM) accounts for about $90 \%$ of diabetes mellitus, for its complexity. ${ }^{3,4}$ T2DM is usually caused by insulin resistance (IR), which contributed to promoting glucose 
production in the liver and reduced glucose uptake in muscle and adipose tissue at a set insulin level. ${ }^{5}$ The development of T2DM involves many stages, including glucose tolerance impairment, insulin resistance, hyperinsulinemia, hyperglycemia, and insulin deficiency in most patients. ${ }^{6}$ However, insulin resistance and relative insulin deficiency have been considered essential to the etiology of T2DM. Therefore, insulin resistance has become the chief objective of the T2DM treatment.

Sirt1 is a histone deacetylase. As a multifunctional transcriptional regulator, it is closely related to cell survival, metabolic process and life activities including proliferation, senescence and apoptosis. It is widely involved in regulating multiple signal pathways of mammalian cell lifespan and multiple metabolic pathways such as glucose and lipid metabolism and insulin secretion to maintain the stability of glucose and lipid metabolism. ${ }^{7,8}$ It has been found that Sirt1 is expressed in liver, brain, kidney, fat and muscle tissues, and relatively uniformly expressed only in islet cells. ${ }^{8}$ And the expression of Sirt1 in the pancreas of mammals is significantly upregulated under the condition of calorie restriction (CR $)^{9}$ When insulin resistance occurs in the early stage of T2DM, with the damage of $\beta$ cells, the Sirt1 in the cells is significantly decreased. In addition, in the obese mice model of insulin resistance induced by high-fat (HF) or high-sucrose, the expression of Sirt1 in the pancreas decrease significantly. ${ }^{10}$ Therefore, Sirt1 may be a novel therapeutic target for the treatment of insulin resistance and T2DM.

Roux-en-Y gastric bypass (RYGB) is the most effective treatment to reduce body weight and improve glycemic status including T2DM. ${ }^{11,12}$ The weight loss and improvement of glycemic status after RYGB caused by RYGB induced rerouting of nutrients and improved the secretion of gastrointestinal hormones, such as ghrelin, peptide YY and glucagon-like peptide-1. ${ }^{13}$ RYGB helps to hyperinsulinemia and early normalization of glucose levels by promoting the hypersecretion of GLP-1 in T2DM. ${ }^{14}$ However, the role and molecular mechanism of RGYB on liver glucose metabolism are unclear. In conclusion, we speculate that Sirt1 may be involved in the therapeutic effect of RGYB on liver glucose metabolism and play a promoting role.

The objective of this study was to assess the pathological changes of liver, insulin resistance and the glucose metabolism after RYGB in rats. Further, we aimed to investigate the molecular mechanism of RGYB on liver glucose metabolism.

\section{Materials and Methods Cell Culture}

Human hepatic immortalized cell lines (FL62891 and HHL5) were obtained from the Shanghai Cell Bank of the Chinese Academy of Sciences, China. Cells were cultured in DMEM supplemented with $10 \%$ fetal bovine serum (FBS, Hyclone) and incubated in an incubator with $5 \% \mathrm{CO}_{2}$ at $37^{\circ}$ C. The cells were passaged once every 3 days. Cells in logarithmic phase were used for the next study.

The insulin resistance models of liver cells FL62891 and HHL5 were treated with $10^{-7} \mathrm{~mol} / \mathrm{L}$ human recombinant insulin and cultured for $24 \mathrm{~h}$ in an incubator with 5\% $\mathrm{CO}_{2}$ at $37^{\circ} \mathrm{C}$. The insulin-resistant FL62891 and HHL5 cells have biological effects of insulin resistance.

\section{Cell Transfection}

The insulin-resistant FL62891 and HHL5 cells were seeded at a density of $2 \times 10^{5}$ cells $/ \mathrm{mL}$ in 6 -well plates. The plasmid of Sirt1 was used for Sirt1 overexpression. Then, the overexpression plasmid of Sirt1 (pcDNA-Sirt1, 500ng/ $\mu$, GenePharma, Shanghai, China) and negative control (pcDNA-NC, GenePharma) were incubated in Opti-MEM (Invitrogen, Carlsbad, USA) using Lipofectamine $^{\mathrm{TM}} 2000$ (Invitrogen, Carlsbad, USA) according to the manufacturer's protocol. The inhibitor NT157 of IRS1 (3 $\mu$ M, Beijing Bio Lebo Technology Co., Ltd, Beijing, China) was added in FL62891 and HHL5 cells. ${ }^{15}$

\section{Hematoxylin and Eosin (H\&E) and Masson Staining}

Liver tissues were fixed with $10 \%$ buffered formalin at room temperature for $48 \mathrm{~h}$. They are then dehydrated and embedded in paraffin. The sections were stained with hematoxylin and eosin H\&E Masson's trichrome for histological evaluation. Blinded analysis of liver tissues was performed using a light microscope (Leica DM 500, Leica light microscope, Wetzlar, Germany). Moreover, tumor tissues pathological alterations were examined by a boardcertified pathologist.

\section{Immunohistochemistry Assay}

For immunohistochemistry analysis, the fresh tumor tissue samples were fixed in formalin for $48 \mathrm{~h}$. Then, the tissue 
block was put into paraffin and cut into slides for the desired thickness in a microtome. Next, it was fixed into a slide. After washing, the samples were prepared for blocking and incubating with antibody which were diluted in $5 \%$ horse serum with chilled PBS at $4{ }^{\circ} \mathrm{C}$ overnight. Isotype-matched IgG was used instead of primary antibody against Sirt1 (1:1000; ab189494; Abcam) as a negative control of the staining. Sections were then incubated with diluted streptavidin-peroxidase HRP at room temperature with a staining kit, according to the manufacturer's instructions. The sections were then stained with hematoxylin for $5 \mathrm{~min}$ and were mounted and observed using a phase-contrast microscope.

\section{Immunofluorescence}

After treatment, the cells were fixed with acetone:methanol (1:1). The slides were washed with PBS/0.05\% Tween 20 at room temperature (RT), and blocked with serum-free DAKO-Block (DAKO Deutschland GmbH, Hamburg, Germany). Then, it was washed again and was incubated with 1:1000 primary antibodies against FGF-21 (ab171941; Abcam) and PTP1B (ab244207; Abcam). After washing, the slides were incubated with second antibody, and they were washed again and were incubated with 4',6-diamidino-2-phenylindole (DAPI; Invitrogen). Finally, the slides were washed, mounted with fluorescence mounting medium (DAKO) and analyzed using a Zeiss Axiovert 200 microscope (Zeiss) with the VisiView software (Visitron Systems, Puchheim, Germany).

\section{In vivo Mouse Experiments}

SD rats of SPF grade, aged 5-6 weeks, were purchased from Beijing HFK BIOSCIENCE CO., LTD (Beijing, China). Rats were kept in an SPF environment at 20-24 $\mathrm{C}$ with $50-60 \%$ humidity in cycle of light $12 \mathrm{~h}$ and dark 12h. After adaptation to the environment and being fed for 2 weeks. Twenty-four SD rats were randomly divided into four groups: control, DM, Diet, RYGB. The 12 rats in DM and RYGB were free access to food and water. They were subjected to intraperitoneal injection of STZ $30 \mathrm{mg} / \mathrm{kg} 4$ weeks later, and continued to be fed with high-fat diet. The blood glucose level was recorded weekly. When the random blood glucose was $16.7 \mathrm{mmol} / \mathrm{L}$ and remained stable for 4 weeks, the rats were identified as Type 2 diabetes. ${ }^{16}$ The rats were fasted for $12 \mathrm{~h}$ before surgery, and were anesthetized with isoflurane inhalation, then supine on the operating table.
Rats in the RYGB operation group were made a longitudinal incision along the midabdominal line of $3 \mathrm{~cm}$, and the proximal end of the stomach was cut off with an arc under the cardia to form $20 \%$ gastric sac, and the distal residual stomach was closed with 6-0 suture line. The jejunum was dissected about $15 \mathrm{~cm}$ from the distal end of the Treitz ligament, and the distal jejunum was anastomosed to the gastric sac. Proximal and distal jejunum anastomoses were performed at $10 \mathrm{~cm}$ from the anastomosis. The rats were given $10 \%$ glucose solution for 3 days. At the end of the experiment, the liver tissues were harvested and stored in RNAlater ${ }^{\mathrm{TM}}$ stabilization solution for downstream studies. All the above experimental operations have been approved by the ethics committee of Yixing People' s Hospital.

\section{qRT-PCR Analysis}

Total RNAs were extracted from cells by TRIzol reagent (Thermo Fisher Scientific, Inc.). RNAs were synthesized into cDNA using a reverse transcription kit (Promega). PCR was performed using SYBR green Supermix (ABI) in ABI 7500 PCR system. PCR conditions were as follows: $94{ }^{\circ} \mathrm{C}$ for $30 \mathrm{sec}, 55^{\circ} \mathrm{C}$ for $30 \mathrm{sec}$ and $72{ }^{\circ} \mathrm{C}$ for $30 \mathrm{sec}$ ( 32 cycles). The relative expressions of target genes were calculated by $2^{-\Delta \Delta} \mathrm{Ct}$ method and normalized to the housekeeping gene GAPDH. The sequences of PCR primers were as follows: Sirt1 forward, 5'-TGACCGATGGACTCCTCACT-3' and reverse, 5'-ATTGTTCGAGGATCGGTGCC-3'; GAPDH forward, 5'-TGACCTCAACTACATGGTCTACA-3' and reverse, 5'-CTTCCCATTCTCGGCCTTG-3'.

\section{Western Blotting Assay}

Total protein was extracted from cells and liver tissues using RIPA buffer. Protein concentrations were determined with a Pierce BCA Protein Assay Kit (Pierce Biotechnology, Rockford, IL). Next, 20 ug protein was loaded and separated with $12 \%$ SDS gels and transferred to polyvinylidene difluoride membrane (Millipore, Bedford, MA). Following transfer, membranes were blocked with $10 \%$ skimmed milk and incubated with the appropriate 1:1000 primary antibodies against Sirt1 (ab189494, Abcam), FGF-21 (ab171941, Abcam), PTP1B (ab244207, Abcam), p-PKB (ab175349, Abcam), p-mTOR2 (ab134903, Abcam), IRS1 (ab40777, Abcam), GLUT4 (ab654, Abcam) and GAPDH (ab9485, Abcam) at $4^{\circ} \mathrm{C}$ overnight. Furthermore, the corresponding HRP-conjugated secondary antibodies were used to detect the protein expression. Chemiluminescent film (Roche) was applied for assessment of protein expression with ImageJ software (NIH). 


\section{Statistical Analysis}

The SPSS13.0 statistical software was used to analyze the results, and the measurement data were expressed as the mean \pm standard deviation. The single-factor analysis of variance was applied to compare the intergroup difference. $\mathrm{P}<0.05$ was considered statistically significance difference.

\section{Results}

\section{Following RYGB Improved the}

Pathological Changes of Liver in Rats with Type 2 Diabetes Mellitus

To investigate the potential roles of RYGB in rats with type 2 diabetes mellitus, H\&E staining assay was performed to explore the pathological changes of liver. As shown in Figure 1A, the rat hepatocytes were arranged in a cord-like and orderly manner with the central vein as the center. In DM group, hepatocytes have steatoid degeneration. The cells around the central vein are filled with fat droplets of different sizes. The cord-like structure is irregular. In Diet group, hepatocyte steatosis was slightly improved. After RYGB treatment, the hepatocytes showed mild steatosis and the cord-like structure of sinus hepaticus was regular. Additionally, Masson staining assay was employed to explore the changes of liver fibrous tissue. As shown in Figure 1B, no obvious staining of fibrous tissue was observed in NC group.
The staining of green stained fibrous tissue was increased in the porta hepatis of DM rats. The green staining area in Diet group was decreased and the highdose group was the most significant. Therefore, following RYGB improved the pathological changes of liver in rats with type 2 diabetes mellitus.

\section{The Expression of Sirtl Was Upregulated in Following RYGB in Rat Liver with Type 2 Diabetes Mellitus}

To further explore the molecular mechanism of following RYGB improved the pathological changes of liver in rats with type 2 diabetes mellitus, we validated the protein level expression of Sirt1 in rat liver with type 2 diabetes mellitus. The protein expression of Sirt1 was measured in Control, DM, Diet and RYGB groups by Western blotting. As shown in Figure 2A, the protein expression of Sirt1 was reduced in the DM group, whereas the protein expression of Sirtl was increased in Diet and RYGB groups. Moreover, the protein expression of Sirt1 in RYGB group was higher than that of Diet group, showing statistically significant difference, compared to the Control group. Equally, the protein expression of Sirt1 revealed by immunohistochemical assay was similar to the result of Western blotting (Figure 2B). Thus, the expression of Sirt1 was upregulated in following RYGB in rat liver with type 2 diabetes mellitus.
A

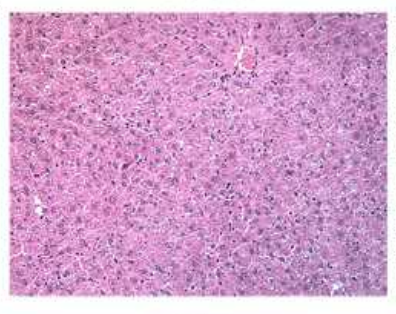

Diet

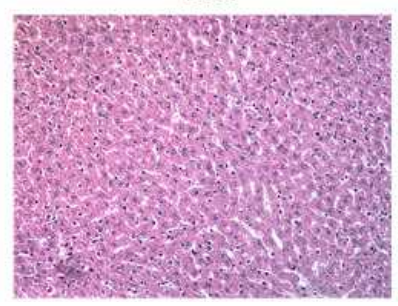

DM

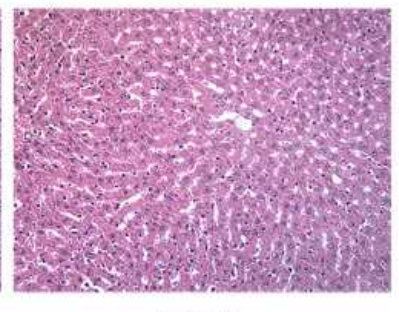

RYGB

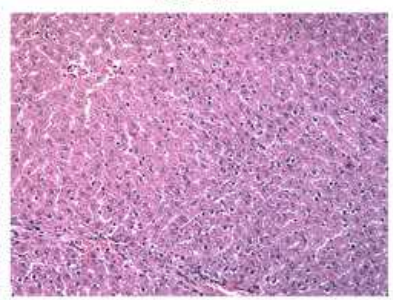

B

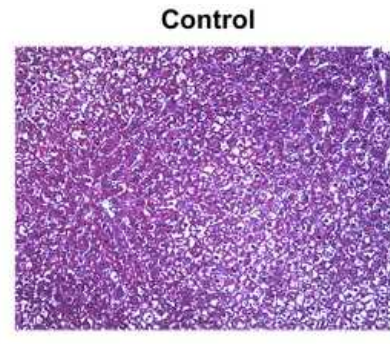

Diet

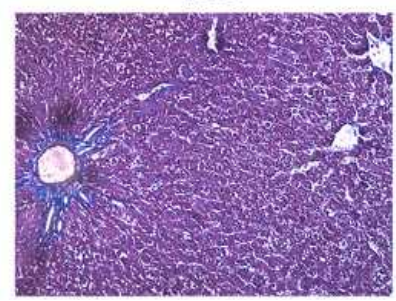

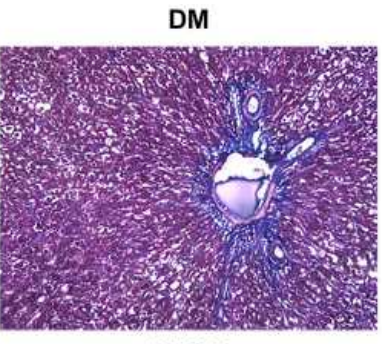

RYGB

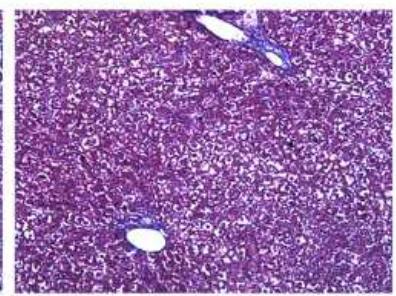

Figure I Following RYGB improved the pathological changes of liver in rats with type 2 diabetes mellitus. (A) H\&E staining assay was explored to the changes of liver fibrous tissue. (B) Masson staining assay was explored to the changes of liver fibrous tissue. 
A
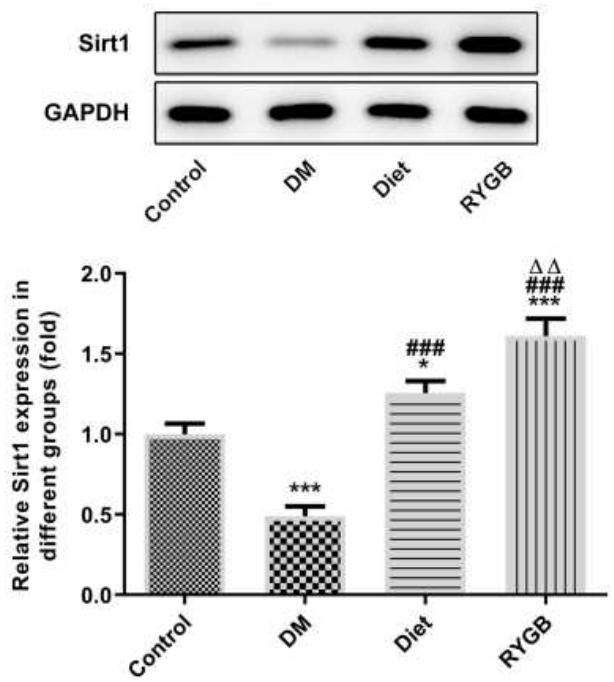

B

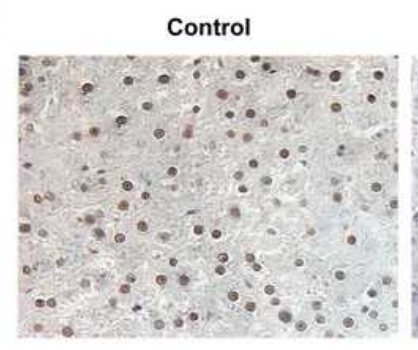

Diet

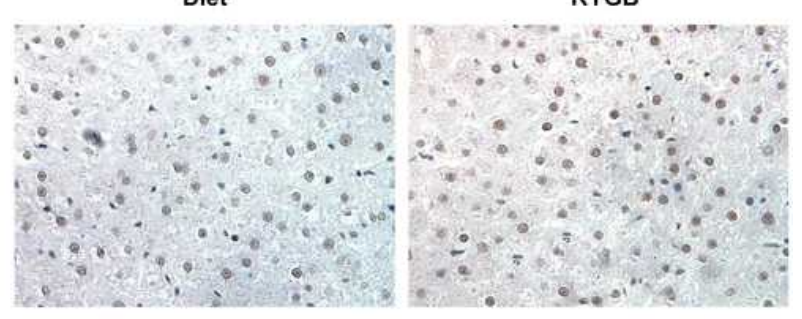

Figure 2 The expression of Sirt I was upregulated in following RYGB in rat liver with type 2 diabetes mellitus. (A) The protein expression of Sirt I was detected in Control, DM, Diet and RYGB groups by Western blotting. (B) Immunohistochemical assay was performed to detect the expression of Sirtl. *p $<0.05$ and ***p $<0.00$ I vs Control; \#\# $<0.00$ I vs DM; ${ }^{\Delta \Delta} \mathrm{p}<0.0$ I vs Diet.

Effect on the Expressions of FGF-2I and PTPIB, and the Activation of IRSI/ mTOR2/PKB Pathway in Following RYGB in Rat Liver with Type 2 Diabetes Mellitus

To evaluate the effects of following RYGB on the expressions of metabolic regulatory molecules FGF-21 and PTP1B in rat liver with type 2 diabetes mellitus, the expressions of FGF-21 and PTPB1 were detected by Western blotting and immunofluorescence. As shown in Figure 3A, results of Western blot showed that the expression of FGF-21 was decreased in DM group and increased in Diet group, and RYGB significantly promoted the expression of FGF-21 compared with Control group, while the expression of FGF-21 was the highest in RYGB group than that in Diet group with statistical difference compared to the Control group. Meanwhile, the expression of PTP1B was increased in DM group and was decreased in Diet group and RYGB group compared with Control group, whereas the expression of FGF-21 was lower in RYGB group than Diet group, showing statistically significant difference compared with the Control group. Next, results of immunofluorescence assay showed that RYGB and Diet significantly increased the expression of FGF-21 and decreased the expression of PTP1B in rats with type 2 diabetes mellitus, there was a statistical difference between DM group and RYGB group (Figure 3B and C). These phenomenon indicated that following RYGB had effects on the expression of FGF-21 and PTP1B. Next, the phosphorylation levels of IRS1/mTOR2/PKB pathway-related proteins (PKB, mTOR2, IRS1 and GLUT4) were detected by Western blotting. GLUT4 in liver tissue of DM rats showed low expression, which was involved in promoting glucose utilization. ${ }^{17}$

As shown in Figure 3D, RYGB and Diet significantly increased the expressions of p-PKB, p-mTOR2, IRS1 and GLUT4 compared to the DM group, and the expressions of p-PKB, p-mTOR2, IRS1 and GLUT4 was the highest in RYGB group. Thus, following RYGB could regulate the glucose metabolism through IRS1/mTOR2 /PKB pathway in rats with type 2 diabetes mellitus.

\section{The Expression of Sirt I Was Downregulated and the Effect of Sirtl Overexpression on the Expressions of FGF-2I and PTPIB in \\ Insulin-Resistant FL6289I and HHL5 Cells}

Subsequently, we detected the expression of Sirt1 in insulin-resistant cells. The insulin resistance model of liver cells FL62891 and HHL5 was built using human recombinant insulin, as. As shown in Figure 4A, compared with the untreated FL62891 cells, Sirt1 was downregulated in insulin-resistant FL62891 cells. The expression of Sirt1 in insulin-resistant FL62891 cells 

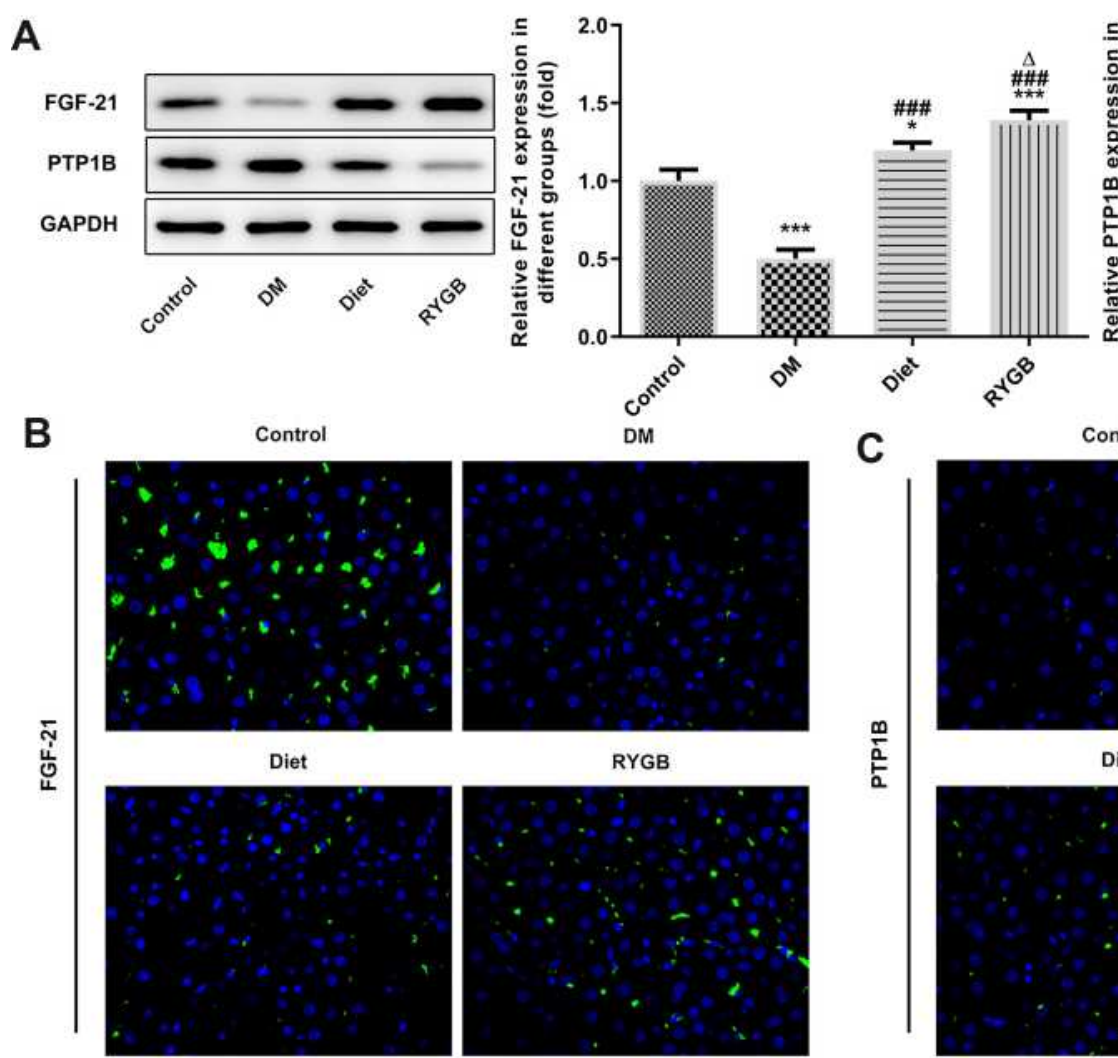

C
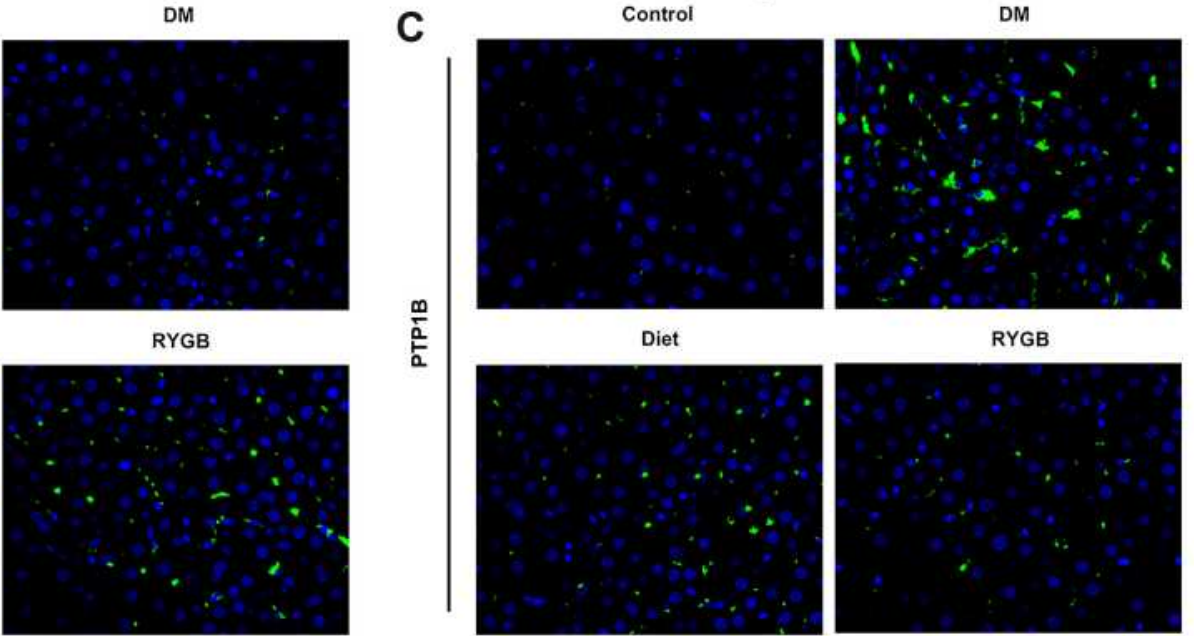

$\frac{\Sigma_{0}^{2}}{2}$

Diet

RYGB
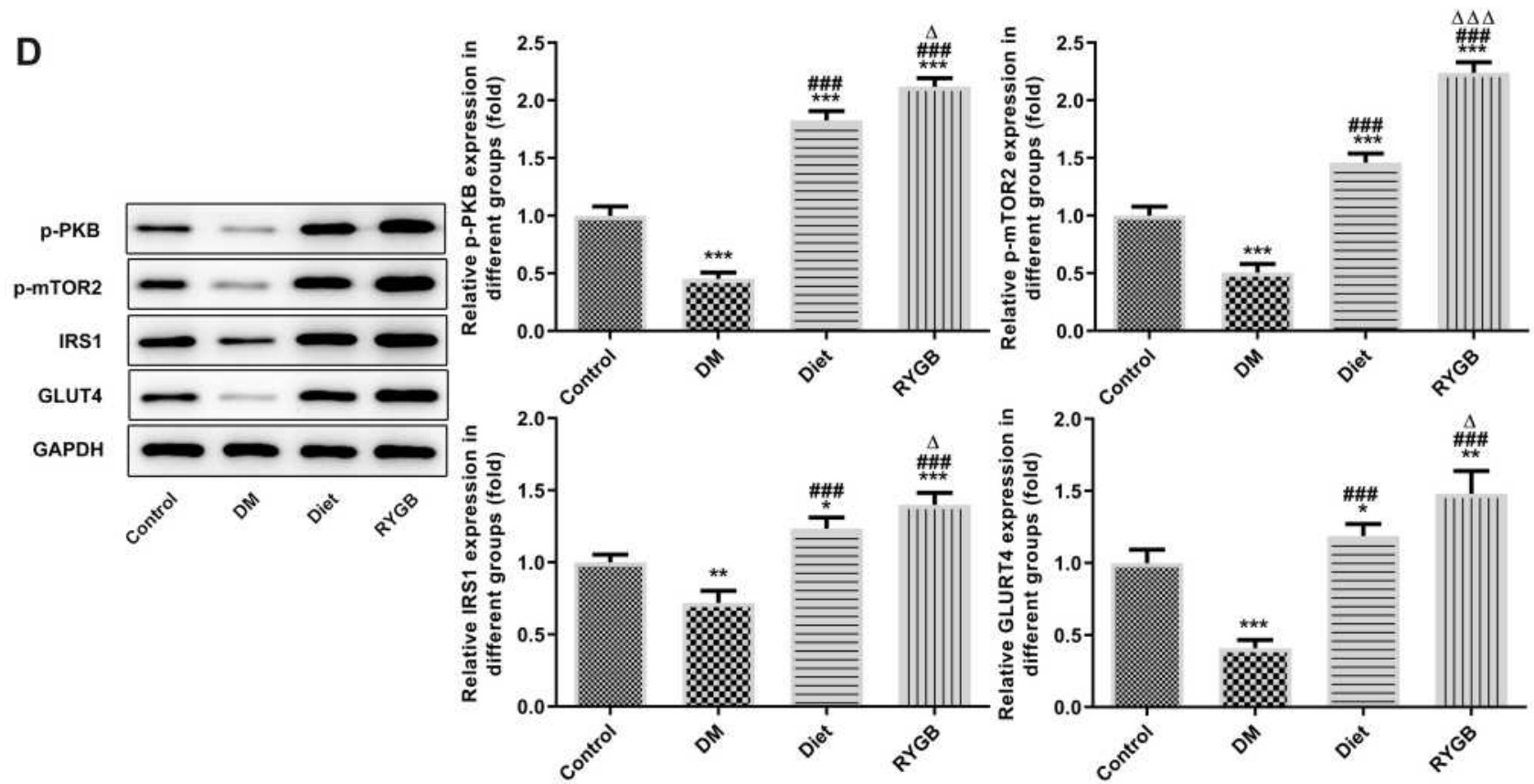

Figure 3 The effect on the expressions of FGF-2I and PTPIB, and the activation of IRSI/mTOR2 /PKB pathway in following RYGB in rat liver with type 2 diabetes mellitus. (A) The effect on the expressions of FGF-2I and PTPIB in following RYGB in rat liver with type 2 diabetes mellitus was detected by Western blotting. (B) The effect on the expressions of FGF-2I in following RYGB in rat liver with type 2 diabetes mellitus was detected by immunofluorescence analysis. (C) The effect on the expressions of PTPIB in following RYGB in rat liver with type 2 diabetes mellitus was detected by immunofluorescence analysis. (D) The effect on the expressions of IRSI/mTOR2 /PKB pathwayrelated proteins (p-PKB, p-mTOR2, IRSI and GLUT4) in rat liver with type 2 diabetes mellitus was detected by Western blotting. ${ }^{*} p<0.05$, ${ }^{*} \mathrm{p}<0.01$ and $* * * \mathrm{p}<0.00 \mathrm{I}$ vs Control; ${ }^{\#} \mathrm{p}<0.001$ vs DM; ${ }^{{ }} \mathrm{p}<0.05,{ }^{\Delta \Delta} \mathrm{p}<0.01$ and ${ }^{\Delta \Delta \Delta} \mathrm{p}<0.001$ vs Diet. 
A
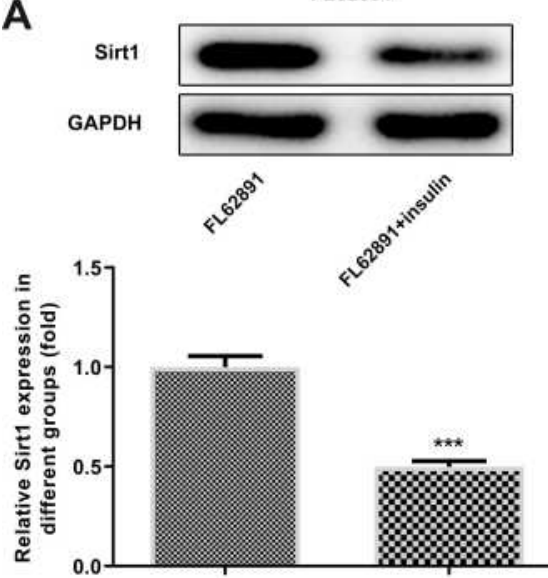

C

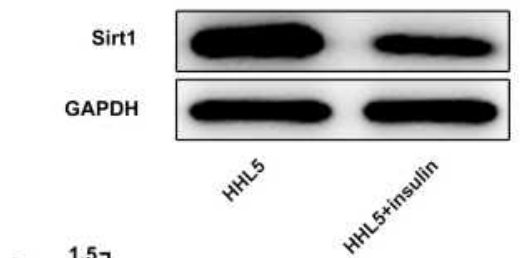

B
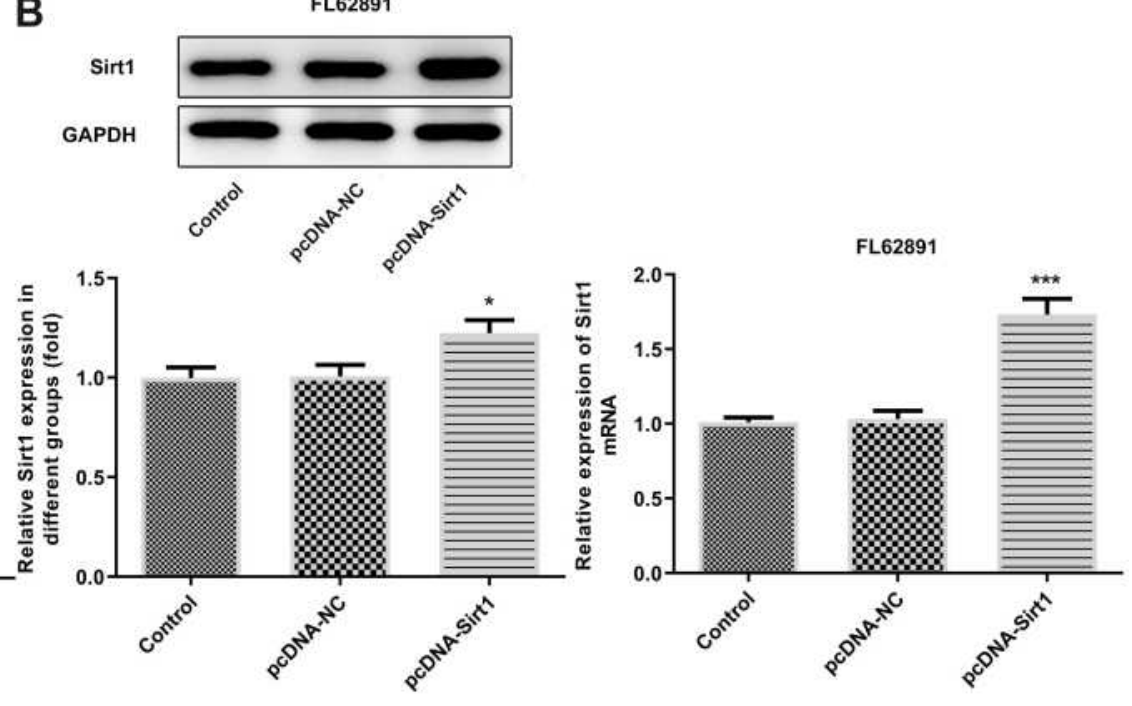

D
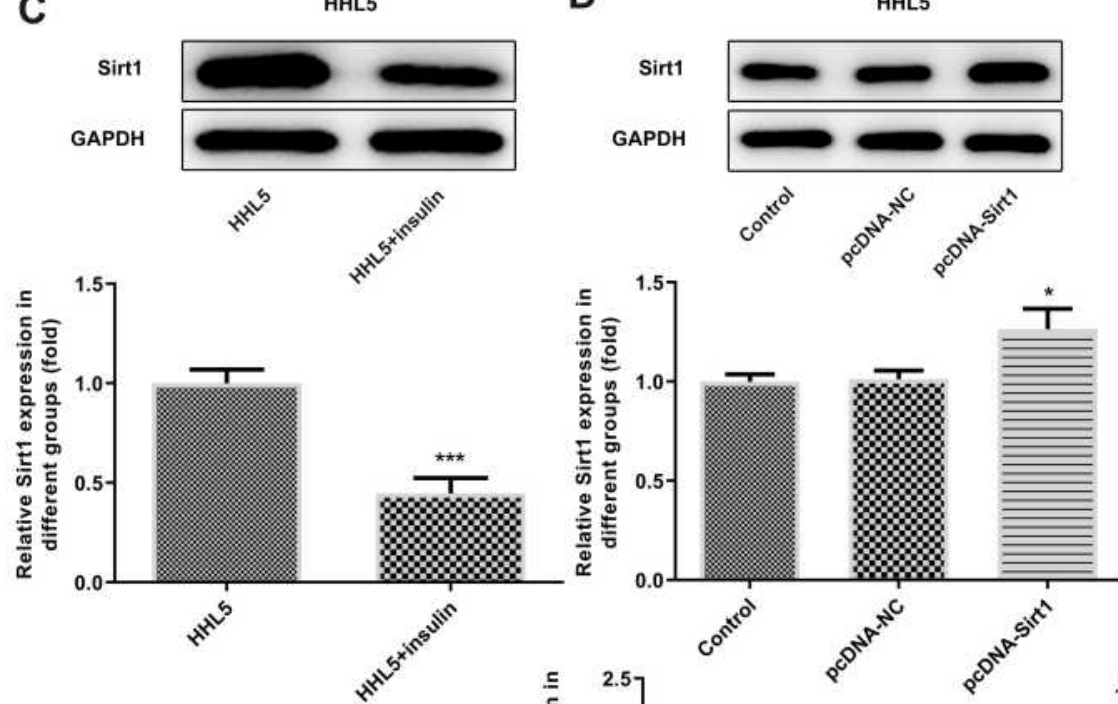

E

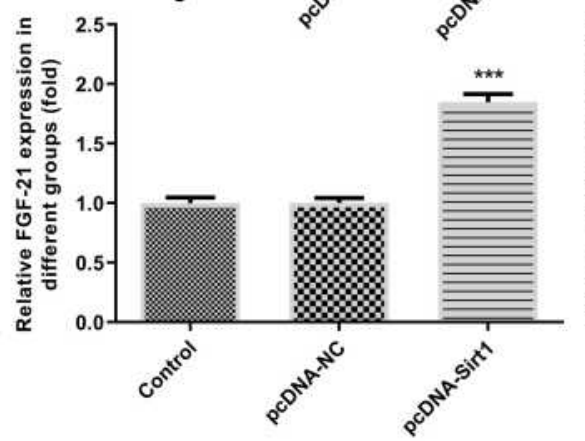

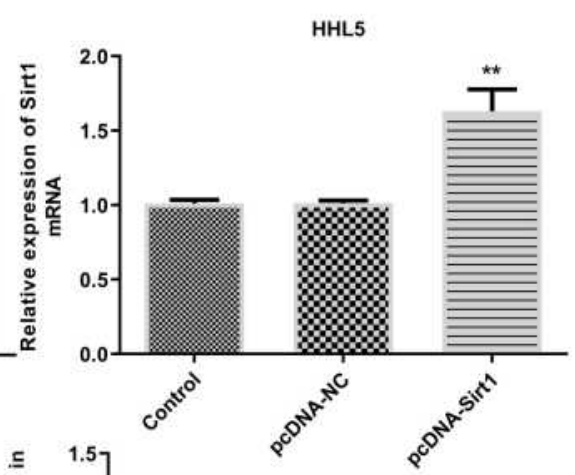

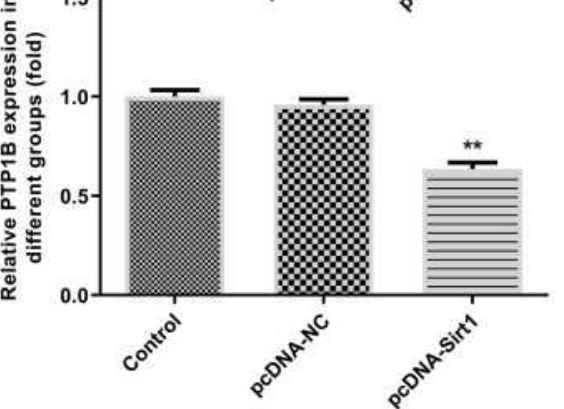

Figure 4 The expression of Sirt I was downregulated and the effect of Sirtl overexpression on the expressions of FGF-2I and PTPIB in insulin resistance FL6289I and HHL5 cells. (A) The expressions of Sirtl in the untreated FL6289I cells and insulin resistance FL6289I cells were detected by Western blotting. *** $<0.00$ I vs FL6289I. (B) The efficiency of Sirtl overexpression was examined by qRT-PCR and Western blotting in transfected insulin resistance FL6289I cells. *p $<0.05$ and $* * * p<0.001$ vs pcDNA-NC. (C) The expressions of Sirtl in the untreated HHL5 cells and insulin resistance HHL5 cells were detected by Western blotting. *** $<$ < $0.00 \mathrm{I}$ vs HHL5. (D) The efficiency of Sirtl overexpression was examined by qRT-PCR and Western blotting in transfected insulin resistance HHL5 cells. *p $<0.05$ and $* * *$ p $<0.00$ I vs pcDNA-NC. (E) Effects of Sirtl overexpression on the expressions of FCF-2I and PTPIB in insulin resistance FL6289I and HHL5 cells. **p $<0.01$ and ***p $<0.00 \mathrm{I}$ vs pcDNA-NC.

was increased by the transfection with pcDNA-Sirt1.

As shown in Figure 4B, the results of Western blot and qRT-PCR showed that the expression of Sirt1 in pcDNA-Sirt1 group was significantly higher than that in pcDNA-NC group. Similar results were observed in insulin-resistant HHL5 cells. As shown in Figure 4C, compared with the untreated HHL5 cells, Sirt1 was downregulated in insulin-resistant HHL5 cells. The 
expression of Sirt1 in insulin-resistant HHL5 cells was increased by the transfection with pcDNA-Sirt1. As shown in Figure 4D, the results of Western blot and qRT-PCR showed that the expression of Sirt1 in pcDNA-Sirt1 group was significantly higher than that in pcDNA-NC group. Finally, the effects of Sirt1 overexpression on the expressions of FGF-21 and PTP1B in FL62891 and HHL5 cells were detected by Western blotting. As shown in Figure 4E, Sirt1 overexpression significantly increased the expression of FCF-21, whereas it decreased the expression of PTP1B compared with the pcDNA-NC group. Taken together, the expression of Sirt1 was downregulated and sirt1 exerted its effects on the expression of FGF-21 and PTP1B in insulin-resistant FL62891 and HHL5 cells.

\section{Effect of Sirtl Overexpression on the Expression of FGF-2I and PTPIB in Insulin-Resistant FL6289I and HHL5 Cells}

In order to detect the effect of Sirtl overexpression on the expressions of FGF-1 and PTP1B again, this effect was verified by immunofluorescence. As shown in Figure 5A and B, Sirt1 overexpression promoted the expression of FCF-21 and reduced the expression of PTP1B in insulin-resistant FL62891 cells. In addition, in insulin-resistant HHL5 cells, Sirt1 overexpression could also promote the expression of FCF-21 and suppressed the expression of PTP1B (Figure 5C and D). These results demonstrated that Sirt1 exerted its effects on the expression of FGF-21 and PTP1B in insulin-resistant FL62891 and HHL5 cells.
A FL62891

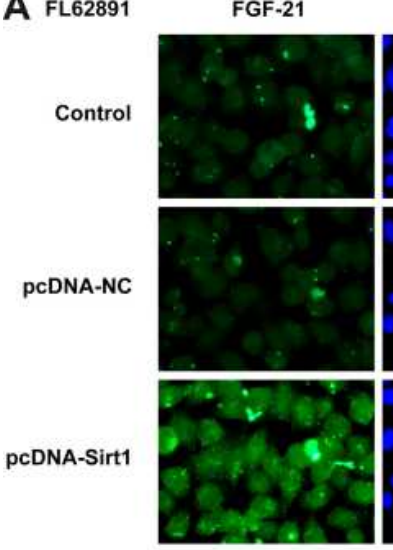

C HHL5

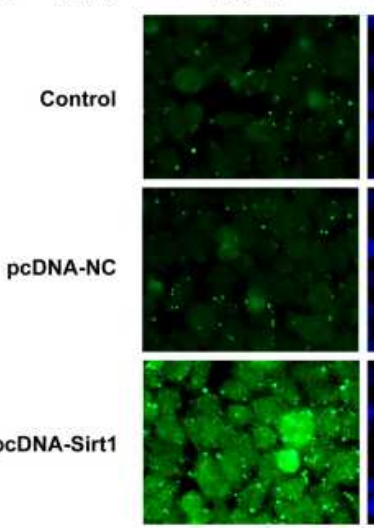

DAPI

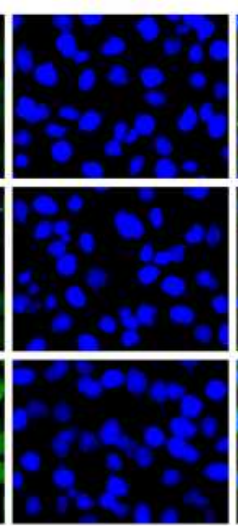

DAPI

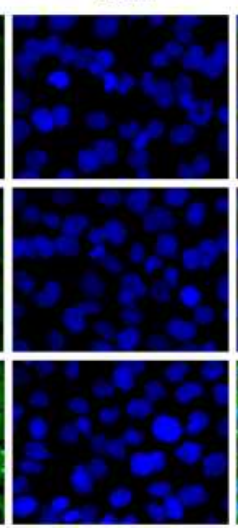

Merged

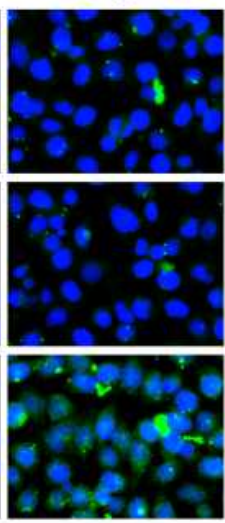

Merged

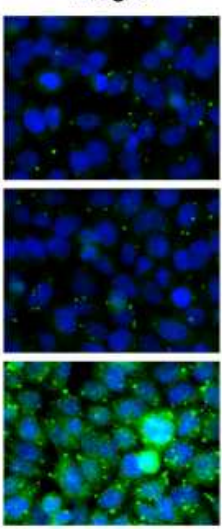

B FL62891

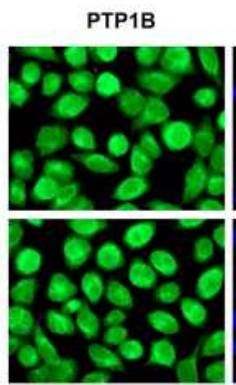

Control

pcDNA-NC

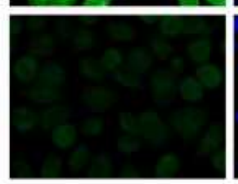

D HHL5

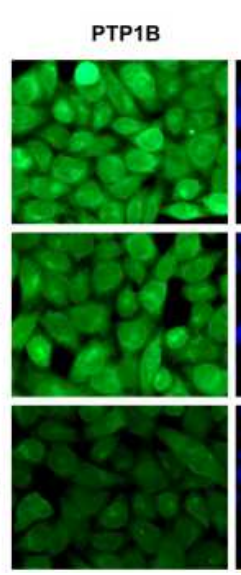

DAPI

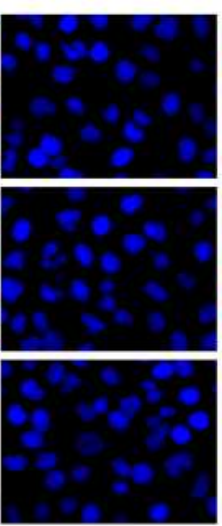

DAPI

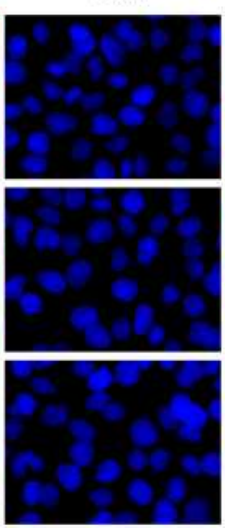

Merged

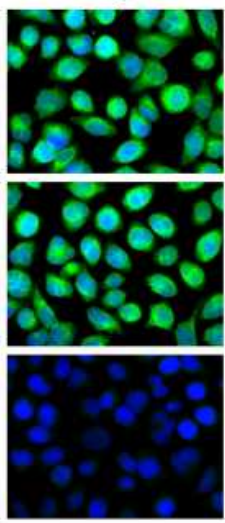

Merged

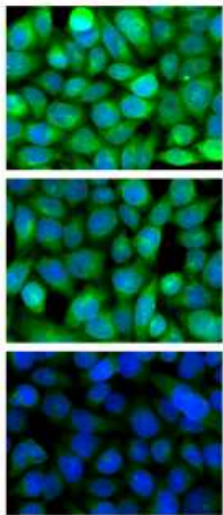

Figure 5 The effect of Sirt I overexpression on the expression of FGF-2I and PTPIB in insulin resistance FL6289I and HHL5 cells. (A) The effect of Sirt I overexpression on the expression of FGF-2I in insulin resistance FL6289I cells was detected by immunofluorescence. (B) The effect of Sirt I overexpression on the expression of PTPBI in insulin resistance FL6289 I cells was detected by immunofluorescence. (C) The effect of Sirt l overexpression on the expression of FCF-2I in insulin resistance HHL5 cells was detected by immunofluorescence. (D) The effect of Sirtl overexpression on the expression of PTPBI in insulin resistance HHL5 cells was detected by immunofluorescence. 


\section{Sirtl Regulated the Glucose Metabolism Through IRSI/mTOR2 /PKB Pathway in Insulin-Resistant FL6289I and HHL5 Cells}

In the end, effects of Sirt1 overexpression on the activation of IRS1/mTOR2 /PKB pathway were detected by Western blotting. As shown in Figure 6A and B, Sirt1 overexpression significantly increased the expressions of IRS1 and GLURT4, and the phosphorylation levels of PKB and mTOR2 compared with the Control groups in insulinresistant FL62891 and HHL5 cells. To investigate the molecular mechanism of Sirt1 on the glucose metabolism, we added the inhibitor NT157 of IRS1 $(3 \mu \mathrm{M})^{18}$ in insulinresistant FL62891 and HHL5 cells, and divided the experimental groups into Control+NT157, pcDNA-Sirt1+NT157 and pcDNA-Sirt1+NT157. As shown in Figure $6 \mathrm{C}$ and D, results of Western blotting revealed that the Sirt1 overexpression decreased the expressions of IRS1 and GLURT4, the phosphorylation levels of PKB and mTOR2. These above changes could be reversed by the NT157. Therefore, Sirt1 could regulate the glucose metabolism via IRS1/mTOR2 / PKB pathway in insulin-resistant FL62891 and HHL5 cells.

\section{Discussion}

A large number of clinical researches confirmed that RYGB is an effective surgical treatment for T2DM. ${ }^{19,20}$ The typical RYGB improved the glucose tolerance and insulin sensitivity, along with weight loss and decreased food intake in T2DM. ${ }^{21}$ RYGB decreased visceral fat area correlated with improved arterial stiffness in Chinese obese patients with $\mathrm{T}_{2} \mathrm{DM}{ }^{22,23}$ In the present study, the expression of Sirt1 was upregulated in following RYGB in rat liver with T2DM and the overexpression of Sirt1 significantly increased the expression of FCF-21 whereas decreased the expression of PTPB1 in insulin-resistant FL62891 and HHL5 cells, which may relate to the IRS1/mTOR2/PKB pathway.

Silent information regulator 1 (Sirt1) is a class of $\mathrm{NAD}^{+}$-dependent protein deacetylase. Previous studies showed that Sirt1 is closely related to multiple pathological links of insulin resistance and the activation of Sirt1 can directly enhance the insulin sensitivity in the liver. ${ }^{25}$ On the one hand, overexpression of Sirt1 regulates the
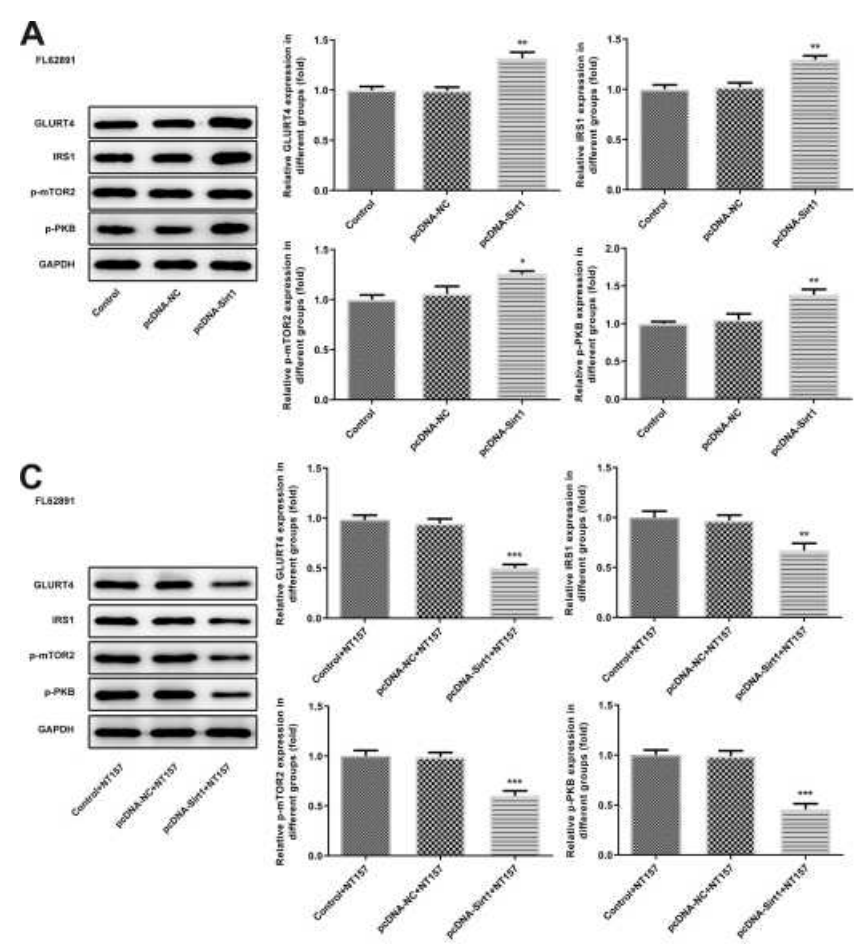
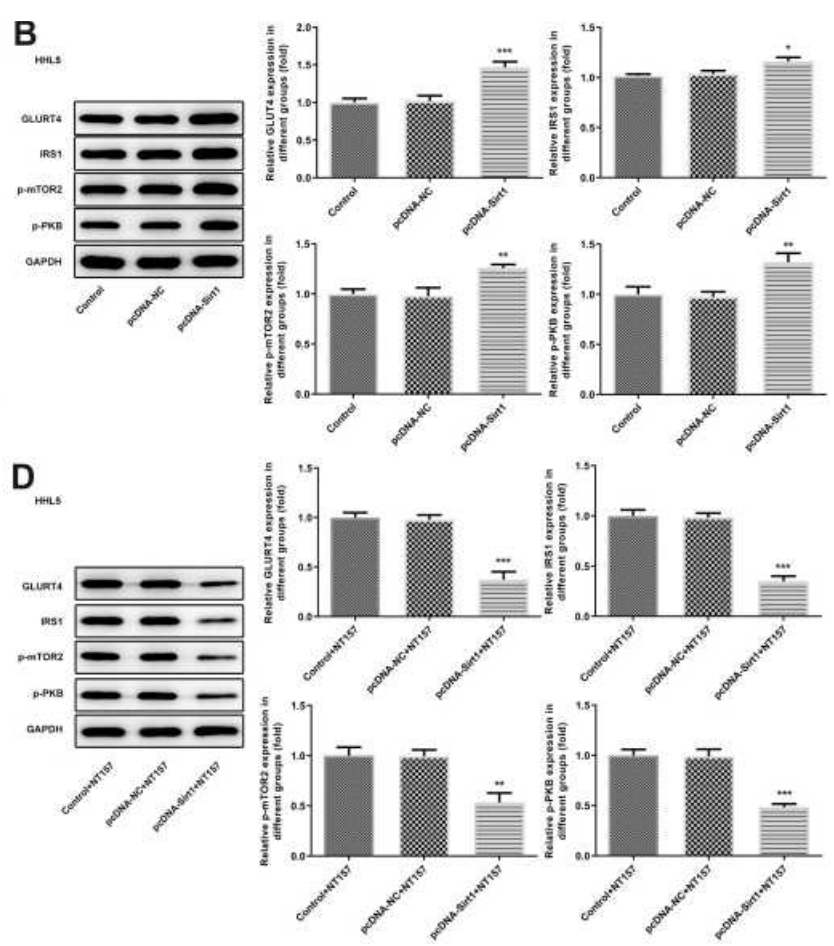

Figure 6 Sirtl regulated the glucose metabolism through IRSI/mTOR2 /PKB pathway in insulin resistance FL6289I and HHL5 cells. (A) Effects of SirtI overexpression on the phosphorylation levels of PKB and mTOR2, and the expressions of IRSI and GLUT4 in insulin resistance FL6289I cells were measured by Western blotting. * $<0.05$ and **p $<0.01$ vs pcDNA-NC. (B) Effects of Sirtl overexpression on the phosphorylation levels of PKB and mTOR2, and the expressions of IRSI and GLUT4 in insulin resistance HHL5 cells were measured by Western blotting. ${ }^{*} p<0.05$, ** $<0.01$ and $*^{* *} p<0.00$ I vs pcDNA-NC. (C) Effects of Sirtl overexpression together with NTI 57 on the phosphorylation levels of PKB and mTOR2, and the expressions of IRSI and GLUT4 in insulin resistance FL6289I cells were measured by Western blotting. **p < 0.01 and ${ }^{* * *} \mathrm{p}<0.001$ vs pcDNA-NC+NTI57. (D) Effects of Sirtl overexpression together with NTI57 on the phosphorylation levels of PKB and mTOR2, and the expressions of IRSI and GLUT4 in insulin resistance HHL5 cells were measured by Western blotting. ${ }^{* *} \mathrm{p}<0.01$ and $* * * \mathrm{p}<0.00 \mathrm{I}$ vs pcDNA-NC+NTI57. 
insulin secretion by inhibiting the expression of mitochondrial uncoupling protein 2 and regulating the sensitivity of $\mathrm{K}^{+}$ions in ATP channel. One previous study showed that Sirt1 can regulate the activity of Sirt1 through deacetylation and then affect the balance of both glucose and lipids in the body. ${ }^{24}$ In addition, the overexpression of Sirt1 improves the insulin resistance in the mouse liver. ${ }^{25}$ Sirt1 knockdown can lead to the oxidative stress and hyperglycemia caused by the overproduction of glucose in the liver. ${ }^{26}$ We found that IRS1 inhibitor could significantly interfere the effects of SIRT1 overexpression. IRS1 is one of the most critical molecules in insulin signaling pathway, and its under-expression or abnormal phosphorylation would affect insulin sensitivity, ${ }^{27}$ which indicated that SIRT1 mediated IR. In general, Sirt1 is expected to be a potential therapeutic target for the improvement of insulin resistance and the treatment in T2DM. In the present study, the expression of Sirt1 is upregulated following RYGB in rat liver with T2DM, whereas is downregulated in insulin-resistant FL62891 and HHL5 cells. This result indicates that Sirt1 may be involved in the treatment of T2DM by RYGB.

Fibroblast growth factor-21 (FGF-21) is a new type of metabolic regulatory molecule, which could bind to the coreceptor $\beta$-Klotho and FGF receptor (FGFRs) to form a relatively stable complex to exert its biological effects. Previous study has shown that FGF21 is a safe, effective and insulin-independent metabolic regulator, which plays an important role in regulating glucose, lipid metabolism and maintaining energy balance in the body. ${ }^{28}$ Smith et al reported that monkeys were injected with different doses of FGF-21 after 6 weeks, their body weight was decreased significantly in a dose-dependent manner. ${ }^{29}$ In addition, FGF-21 could alleviate IR to some degree. ${ }^{30}$ PTP1B is one of the main members in the protein tyrosine phosphatase family, which participates in intracellular signal regulation in multiple tissues and cells, dephosphorylating tyrosine. Protein tyrosine phosphatase-1B (PTP1B) is related to insulin and leptin signal transduction pathway and plays a role in glucose metabolism and energy regulation, which is closely related to the occurrence of T2DM and obesity-related metabolic disorders. It is noteworthy that PTP1B is a negative regulator of insulin signal transduction. ${ }^{31,32}$ An experimental study showed that the blood glucose returned to normal and the weight lost after the obese diabetic mouse model was treated with PTP1B inhibitor. ${ }^{33}$ In the present study, the expression of FGF-21 was upregulated while the expression of PTP1B was downregulated in rat liver with type 2 diabetes mellitus following RYGB. And overexpression of Sirt1 significantly increased the expression of FCF-21 and decreased the expression of PTP1B in insulin-resistant FL62891 and HHL5 cells. Therefore, upregulation of Sirt1 could participate in the process of RYGB treatment of T2DM by promoting FGF-21 and inhibiting PTPB1.

Insulin function is synergistically coordinated by molecular events associated with IR. ${ }^{35} \mathrm{PKB} / \mathrm{mTOR}$ signaling pathway was the major pathway of insulin signaling transduction. ${ }^{34}$ Mammalian target of rapamycin (mTOR) is an important nutrient sensor in T2DM. ${ }^{35}$ In this study, the expression of IRS1 and GLURT4 and the phosphorylation levels of PKB and mTOR2 were increased in RYGB groups compared with the DM group and also exhibited high expression in the pcDNA-Sirt1 group compared with the pcDNA-NC group. Overall, following RYGB could regulate the glucose metabolism through IRS1/mTOR2 /PKB pathways in rats with type 2 diabetes mellitus.

In summary, our studies demonstrated that RYGB improved the pathological changes of liver in rats with T2DM. The expression of Sirt1 was upregulated in following RYGB in rat liver with T2DM. Moreover, Sirt1 overexpression could regulate the glucose metabolism through modulating IRS1/mTOR2 /PKB pathway, and the expressions of FCF-21 and PTP1B in insulin-resistant FL62891 and HHL5 cells. Thus, RYGB improves hepatic glucose metabolism involving in upregulation of Sirt1 in T2DM. This research may provide novel perspective for the treatment of Diabetes mellitus, showing new insight to the effect of RYGB on hepatic glucose metabolism.

\section{Data Sharing Statement}

All data generated or analyzed during this study are included in this published article.

\section{Ethics Approval and Consent}

All animal experiments were approved by the Ethics Medical University and conducted in accordance with the National Institutes of Health (NIH) Guide for the Care and Use of Laboratory Animals.

\section{Acknowledgments}

Not applicable.

\section{Funding}

No funding was received. 


\section{Disclosure}

The authors declare that they have no conflicts of interest for this work.

\section{References}

1. Zheng Y, Ley SH, Hu FB. Global aetiology and epidemiology of type 2 diabetes mellitus and its complications. Nat Rev Endocrinol. 2018;14(2):88-98. doi:10.1038/nrendo.2017.151

2. American Diabetes Association. Classification and diagnosis of diabetes. Diabetes Care. 2015;38(Suppl):S8-s16. doi:10.2337/dc15S005

3. Holman N, Young B, Gadsby R. Current prevalence of Type 1 and Type 2 diabetes in adults and children in the UK. Diabet Med. 2015;32(9):1119-1120. doi:10.1111/dme.12791

4. Bruno G, Runzo C, Cavallo-Perin P, et al. Incidence of type 1 and type 2 diabetes in adults aged 30-49 years: the population-based registry in the province of Turin, Italy. Diabetes Care. 2005;28 (11):2613-2619. doi:10.2337/diacare.28.11.2613

5. Reaven GM. Banting lecture 1988. Role of insulin resistance in human disease. Diabetes. 1988;37(12):1595-1607. doi:10.2337/ diab.37.12.1595

6. Daneshgari F, Liu G, Hanna-Mitchell AT. Path of translational discovery of urological complications of obesity and diabetes. $A m$ J Physiol Renal Physiol. 2017;312(5):F887-f896. doi:10.1152/ ajprenal.00489.2016

7. Rodgers JT, Lerin C, Haas W, Gygi SP, Spiegelman BM, Puigserver P. Nutrient control of glucose homeostasis through a complex of PGC-1alpha and SIRT1. Nature. 2005;434 (7029):113-118. doi:10.1038/nature03354

8. Kume S, Uzu T, Kashiwagi A, Koya D. SIRT1, a calorie restriction mimetic, in a new therapeutic approach for type 2 diabetes mellitus and diabetic vascular complications. Endocr Metab Immune Disord Drug Targets. 2010;10(1):16-24. doi:10.2174/187153010790827957

9. Haigis MC, Mostoslavsky R, Haigis KM, et al. SIRT4 inhibits glutamate dehydrogenase and opposes the effects of calorie restriction in pancreatic beta cells. Cell. 2006;126(5):941-954. doi:10.1016/j. cell.2006.06.057

10. Chen D, Steele AD, Lindquist S, Guarente L. Increase in activity during calorie restriction requires Sirt1. Science (New York, NY). 2005;310(5754):1641. doi:10.1126/science.1118357

11. Li D, Li S, Pan Q, et al. Gastric mammalian target of rapamycin signaling contributes to inhibition of ghrelin expression induced by Roux-en-Y gastric bypass. Cell Physiol Biochem. 2018;51 (2):664-680. doi:10.1159/000495325

12. Ikramuddin S, Korner J, Lee WJ, et al. Roux-en-Y gastric bypass vs intensive medical management for the control of type 2 diabetes, hypertension, and hyperlipidemia: the Diabetes Surgery Study randomized clinical trial. JAMA. 2013;309(21):2240-2249. doi:10.1001/ jama.2013.5835

13. Dirksen C, Jørgensen NB, Bojsen-Møller KN, et al. Gut hormones, early dumping and resting energy expenditure in patients with good and poor weight loss response after Roux-en-Y gastric bypass. Int J Obes. 2013;37(11):1452-1459. doi:10.1038/ijo.2013.15

14. Elahi D, Galiatsatos P, Rabiee A, et al. Mechanisms of type 2 diabetes resolution after Roux-en-Y gastric bypass. Surg Obes Relat Dis. 2014;10(6):1028-1039. doi:10.1016/j.soard.2014.07.011

15. Fenerich BA, Fernandes JC, Apn RA, et al. NT157 has antineoplastic effects and inhibits IRS1/2 and STAT3/5 in JAK2 (V617F)-positive myeloproliferative neoplasm cells. Signal Transduction Targeted Ther. 2020;5(1):5. doi:10.1038/s41392-019-0102-5

16. Li Y, Sun M, Liu Y, Liang J, Wang T, Zhang Z. Gymnemic acid alleviates type 2 diabetes mellitus and suppresses endoplasmic reticulum stress in vivo and in vitro. J Agric Food Chem. 2019;67 (13):3662-3669. doi:10.1021/acs.jafc.9b00431
17. Li S, Huang Q, Zhang L, et al. Effect of CAPE-pNO2 against type 2 diabetes mellitus via the AMPK/GLUT4/ GSK3beta/PPARalpha pathway in HFD/STZ-induced diabetic mice. Eur $J$ Pharmacol. 2019;853:1-10. doi:10.1016/j.ejphar.2019.03.027

18. Rodrigues Alves APN, Fernandes JC, Fenerich BA, et al. IGF1R/ IRS1 targeting has cytotoxic activity and inhibits PI3K/AKT/mTOR and MAPK signaling in acute lymphoblastic leukemia cells. Cancer Lett. 2019;456:59-68. doi:10.1016/j.canlet.2019.04.030

19. Aleassa EM, Hassan M, Hayes K, Brethauer SA, Schauer PR, Aminian A. Effect of revisional bariatric surgery on type 2 diabetes mellitus. Surg Endosc. 2019;33(8):2642-2648. doi:10.1007/s00464018-6541-1

20. Du X, Fu XH, Shi L, Hu JK, Zhou ZG, Cheng Z. Effects of laparoscopic Roux-en-Y gastric bypass on Chinese type 2 diabetes mellitus patients with different levels of obesity: outcomes after 3 years' follow-up. Obes Surg. 2018;28(3):702-711. doi:10.1007/s11695017-2903-3

21. Yan Y, Zhou Z, Kong F, et al. Roux-en-Y gastric bypass surgery suppresses hepatic gluconeogenesis and increases intestinal gluconeogenesis in a T2DM rat model. Obes Surg. 2016;26 (11):2683-2690. doi:10.1007/s11695-016-2157-5

22. Yu H, Chen J, Lu J, et al. Decreased visceral fat area correlates with improved arterial stiffness after Roux-en-Y gastric bypass in Chinese obese patients with type 2 diabetes mellitus: a 12-month follow-up. Surg Obes Relat Dis. 2016;12(3):550-555. doi:10.1016/j. soard.2015.09.003

23. Cottam A, Cottam D, Zaveri H, et al. An analysis of mid-term complications, weight loss, and type 2 diabetes resolution of Stomach Intestinal Pylorus-Sparing Surgery (SIPS) Versus Roux-enY gastric bypass (RYGB) with three-year follow-up. Obes Surg. 2018;28(9):2894-2902. doi:10.1007/s11695-018-3309-6

24. Sin TK, Yung BY, Siu PM. Modulation of SIRT1-Foxo1 signaling axis by resveratrol: implications in skeletal muscle aging and insulin resistance. Cell Physiol Biochem Int J Exp Cell Physiol Biochem Pharmacol. 2015;35(2):541-552. doi:10.1159/000369718

25. Li Y, Xu S, Giles A, et al. Hepatic overexpression of SIRT1 in mice attenuates endoplasmic reticulum stress and insulin resistance in the liver. FASEB J. 2011;25(5):1664-1679. doi:10.1096/fj.10-173492

26. Wang RH, Kim HS, Xiao C, Xu X, Gavrilova O, Deng CX. Hepatic Sirt1 deficiency in mice impairs mTorc2/Akt signaling and results in hyperglycemia, oxidative damage, and insulin resistance. $J$ Clin Invest. 2011;121(11):4477-4490. doi:10.1172/jci46243

27. Copps KD, White MF. Regulation of insulin sensitivity by serine/ threonine phosphorylation of insulin receptor substrate proteins IRS1 and IRS2. Diabetologia. 2012;55(10):2565-2582. doi:10.1007/ s00125-012-2644-8

28. Kolumam G, Chen MZ, Tong R, et al. Sustained brown fat stimulation and insulin sensitization by a humanized bispecific antibody agonist for fibroblast growth factor receptor $1 / \beta$ Klotho complex. EBioMedicine. 2015;2(7):730-743. doi:10.1016/j.ebiom.2015.05.028

29. Smith R, Duguay A, Weiszmann J, et al. A novel approach to improve the function of FGF21. BioDrugs. 2013;27(2):159-166. doi:10.1007/s40259-013-0013-x

30. Geng L, Liao B, Jin L, et al. Exercise alleviates obesity-induced metabolic dysfunction via enhancing FGF21 sensitivity in adipose tissues. Cell Rep. 2019;26(10):2738-2752.e4. doi:10.1016/j. celrep.2019.02.014

31. Zhou J, Wu Z, Oyawaluja BO, et al. Protein tyrosine phosphatase 1B inhibitory iridoids from psydrax subcordata. J Nat Prod. 2019;82 (10):2916-2924. doi:10.1021/acs.jnatprod.9b00770

32. Cho H. Protein tyrosine phosphatase 1B (PTP1B) and obesity. Vitam Horm. 2013;91:405-424.

33. Elchebly M, Payette P, Michaliszyn E, et al. Increased insulin sensitivity and obesity resistance in mice lacking the protein tyrosine phosphatase-1B gene. Science (New York, NY). 1999;283 (5407):1544-1548. doi:10.1126/science.283.5407.1544 
34. Wang S, Wu J, Nie SD, Bereczki E, Pei JJ. Dysregulated mTORdependent signaling in neurodegeneration or carcinogenesis: implication for Alzheimer's disease and brain tumors. J Alzheimer's Dis. 2013;37(3):495-505. doi:10.3233/jad-130641
35. Leibowitz G, Cerasi E, Ketzinel-Gilad M. The role of mTOR in the adaptation and failure of beta-cells in type 2 diabetes. Diabetes Obes Metab. 2008;10(Suppl 4):157-169. doi:10.1111/j.14631326.2008.00952.x

\section{Publish your work in this journal}

Diabetes, Metabolic Syndrome and Obesity: Targets and Therapy is an international, peer-reviewed open-access journal committed to the rapid publication of the latest laboratory and clinical findings in the fields of diabetes, metabolic syndrome and obesity research. Original research, review, case reports, hypothesis formation, expert opinion and commentaries are all considered for publication. The manuscript management system is completely online and includes a very quick and fair peer-review system, which is all easy to use. Visit http://www.dovepress.com/testimonials.php to read real quotes from published authors.

Submit your manuscript here: https://www.dovepress.com/diabetes-metabolic-syndrome-and-obesity-targets-and-therapy-journal 